\title{
Fitness cost of fluoroquinolone resistance in Salmonella enterica serovar Typhimurium
}

\author{
Etienne Giraud, $\uparrow$ Axel Cloeckaert, Sylvie Baucheron, Christian Mouline \\ and Elisabeth Chaslus-Dancla
}

\begin{abstract}
Correspondence
Elisabeth Chaslus-Dancla

chaslus@tours.inra.fr
\end{abstract}

Received 14 January 2003

Accepted 16 April 2003
Unité Bio-Agresseurs, Santé, Environnement, Institut National de la Recherche Agronomique, Centre de Recherche de Tours, 37380 Nouzilly, France

\begin{abstract}
High-level fluoroquinolone (FQ) resistance is still infrequent in salmonellae, compared with other pathogenic enterobacteria. Data provided in this work support the hypothesis that the mechanisms that confer high-level FQ resistance on salmonellae have a prohibitive fitness cost and may thus limit the emergence of highly resistant clones. In vitro mutants that were highly resistant to ciprofloxacin ( $\mathrm{MIC}=8$ and $16 \mathrm{~g} \mathrm{~m} \mathrm{~m}^{-1}$ ) showed generation times 1.4- and 2-fold longer than their parent strains and were unable to colonize the gut of chickens. Electron microscopy showed an altered morphology for one of these mutants grown to stationary phase. Mutants selected in vivo and exhibiting intermediate resistance to ciprofloxacin ( $\mathrm{MIC}=2 \mu \mathrm{g} \mathrm{ml}^{-1}$ ) also showed growth defects on solid media but had normal generation times in liquid culture and colonized the gut of chickens. After in vitro or in vivo passage in the absence of antibiotic selective pressure, partial reversals of the fitness cost were observed, which were associated with slight decreases in resistance to quinolones and other unrelated antibiotics, but were not linked to the loss of gyrA mutations.
\end{abstract}

\section{INTRODUCTION}

The incidence of Salmonella enterica strains resistant to the first-generation quinolone nalidixic acid is increasing. The prevalence of resistance to nalidixic acid is especially high in some serovars such as $S$. enterica serovar Hadar ( $S$. Hadar; 92 and $72 \%$, respectively, in human and animal isolates in 1997 in France) or in some clones such as the epidemic $S$. Typhimurium DT104 in the UK (Breuil et al., 2000; Walker et al., 2000). Nalidixic acid-resistant isolates generally have reduced susceptibility to fluoroquinolones (FQ), which may sometimes compromise treatment (Molbak et al., 1999). However, isolation of strains that exhibit clinical resistance to FQs, as defined by the Comité de l'Antibiogramme de la Société Française de Microbiologie or by the National Committee for Clinical Laboratory Standards, is infrequent. Strains are considered resistant to ciprofloxacin when MICs are $>2 \mu \mathrm{g} \mathrm{ml}^{-1}$ (NCCLS, 1997; Members of the SFM Antibiogram Committee, 2003). In the early 1990s, some $S$. Typhimurium var. Copenhagen isolates, probably epidemiologically related, were described that exhibited high-level resistance to ciprofloxacin $\left(\mathrm{MIC}=32 \mu \mathrm{g} \mathrm{ml}^{-1}\right.$ ) (Heisig, 1993; Heisig et al., 1995). More recently, the emergence of high-level FQ resistance has been reported in S. enterica serotypes Choleraesuis, Schwarzengrund and Typhimurium

†Present address: UMR INRA/ENVN Chimiothérapie Aquacole et

Environnement, Ecole Nationale Vétérinaire de Nantes, Atlanpole - La

Chantrerie, BP 40706, 44307 Nantes Cedex 03, France.

Abbreviation: $\mathrm{FQ}$, fluoroquinolone.
(Chiu et al., 2002; Olsen et al., 2001; Nakaya et al., 2003). However, for the two major human non-typhoidal pathogenic serotypes $S$. Typhimurium and $S$. Enteritidis, the ciprofloxacin MICs remain generally below $1 \mu \mathrm{g} \mathrm{ml}^{-1}$ (Threlfall et al., 2000; Wiuff et al., 2000). Much higher frequencies and levels of resistance are commonly observed for other pathogenic enterobacteria, including Escherichia coli, Klebsiella pneumoniae or Enterobacter cloacae (Chen et al., 2001; Deguchi et al., 1997; McDonald et al., 2001; Paterson et al., 2000; Robert et al., 2001; Weigel et al., 1998). The mechanisms that lead to high-level resistance to FQ in $S$. Typhimurium include alterations in GyrA, the catalytic subunit of DNA gyrase, and overexpression of the AcrAB efflux system (Cloeckaert \& Chaslus-Dancla, 2001; Giraud et al., 1999, 2000; Heisig, 1993; Heisig et al., 1995). Alterations have recently been reported in the ParC catalytic subunit of topoisomerase IV, the secondary target of FQ in high-level FQ-resistant S. Typhimurium (Baucheron et al., 2002; Hansen et al., 2001). We have previously suggested, on the basis of experimental selections, that the mechanisms that confer high-level FQ resistance in $S$. Typhimurium could have an important fitness cost and could therefore be counter-selected under in vivo conditions (Giraud et al., 1999).

The aim of the present study was to characterize further the physiological alterations of FQ-resistant experimental mutants and to investigate whether clones derived from these mutants, still highly resistant to FQ but with better fitness, could emerge in the absence of antibiotic selective pressure. 


\section{METHODS}

Bacterial strains and susceptibility determinations. The parent $S$. Typhimurium strains BN18 and BN82 were respectively isolated from a septicaemic pigeon and from a sick calf. The FQ-resistant mutants BN18/71 and BN82/71 were obtained from these parents, after respectively seven and eight selection steps on Mueller-Hinton agar plates supplemented with increasing concentrations of enrofloxacin (Giraud et al., 1999). BN18/71-R2 and BN82/71-R2 are clones respectively derived from mutants $\mathrm{BN} 18 / 71$ and $\mathrm{BN} 82 / 71$, recovered after about 500 generations in serial transfer cultures in LB medium without antibiotic (Table 1).

Mutants BNJ-529 and BNJ-548 were respectively selected in vivo from strains BN18 and BN82, as described previously (Giraud et al., 1999). Axenic White Leghorn chickens were monocontaminated with either $\mathrm{BN} 18$ or $\mathrm{BN} 82$ and then received increasing doses of enrofloxacin added to the drinking water (from $6 \cdot 25$ to $50 \mu \mathrm{g} \mathrm{ml}^{-1}$ ). Resistant isolates BNJ529 and BNJ-548 were recovered from samples of faeces. BNJ-529/G1 and BNJ-548/G4 were clones respectively derived from mutants BNJ529 and BNJ-548, recovered from the last samples of the colonization experiments described below (Table 1).

MICs were determined by the standard agar doubling dilution method on Mueller-Hinton medium with inocula of $10^{4}$ c.f.u. per spot. The antibiotics used were nalidixic acid, flumequin and penicillin $G$ (Sigma), enrofloxacin and ciprofloxacin (Bayer) and tetracycline and chloramphenicol (Boehringer Mannheim).

Colonization of chickens. One-day-old White Leghorn axenic chickens placed in germ-free isolators were first inoculated with a characterized pathogen-free intestinal flora (Brée et al., 1989). Three days later, they were given per os about $10^{6}$ c.f.u. of one of the following $S$. Typhimurium strains: BN18, BN18/71, BNJ-529, BN82, BN82/71 or BNJ-548. For each strain, we used a group of 15 chickens, placed in a single germ-free isolator. Rectal samples of faeces were taken from five randomly chosen birds on days 1, 3 and 7 after inoculation and then every 7 days for 1 month. Decimal dilutions of the samples were plated on a Salmonella-Shigella medium (SS agar; Sanofi Diagnostics Pasteur), with or without $20 \mu \mathrm{g}$ nalidixic acid $\mathrm{ml}^{-1}$, depending on the phenotype of the inoculated strain (susceptible parent strain or resistant mutant). Salmonella colonies were counted after $18 \mathrm{~h}$ incubation at $37^{\circ} \mathrm{C}$. The limit of detection of the plate counts was $10^{2}$ c.f.u. (g faeces) ${ }^{-1}$. When no Salmonella colonies were recovered from an animal, an enrichment of the undiluted sample was performed in Müller-Kaufman medium (Sanofi Diagnostics Pasteur). In case of positive culture after enrichment, a value of $10^{2}$ c.f.u. ( $\mathrm{g}$ faeces) ${ }^{-1}$ was retained for calculation of the mean. The number of viable Salmonella cells was expressed as $\log _{10}$ c.f.u. (g faeces $)^{-1}$ and means were calculated. Differences in levels of colonization were evaluated by Student's $t$-test.

Monitoring of bacterial growth. The generation times of strains were determined by measuring $\mathrm{OD}_{600}$ in brain heart infusion (BHI). Readings were taken every $30 \mathrm{~min}$ for $8 \mathrm{~h}$. The parent strains systematically included as controls gave similar results throughout the assays. Viable cells were counted by plating every $2 \mathrm{~h}$ during the OD measurements and after $24 \mathrm{~h}$ growth.

Growth of strains was also monitored using an impedance method (Noble et al., 1999). Bacterial suspensions at $2 \times 10^{4}$ c.f.u. $\mathrm{ml}^{-1}$ in $\mathrm{LB}$ medium were distributed in the module wells of a Bactometer microbial monitoring system M-128 (bioMérieux). Changes in capacitance caused by bacterial growth and metabolism were monitored for $18 \mathrm{~h}$. The parameter chosen for assessing the growth of strains was the detection time (DT), defined as the time required by the bacterial population to reach a concentration that caused a rapid deviation from

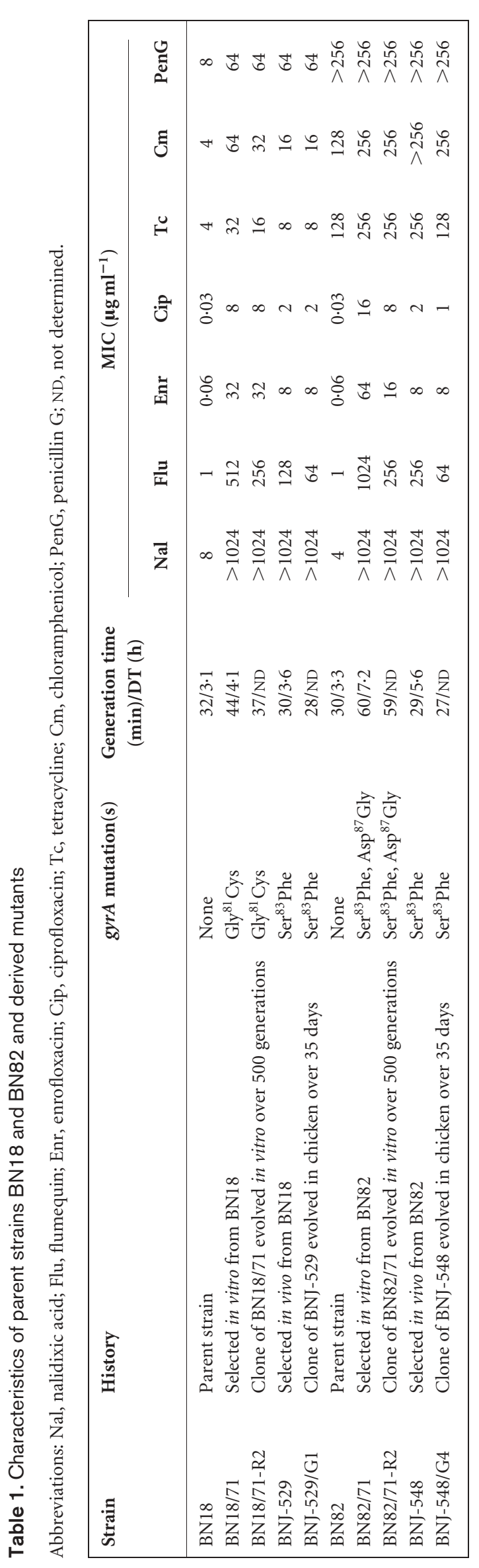


baseline capacitance values. This concentration is approximately of $10^{6}$ c.f.u. $\mathrm{ml}^{-1}$. For a given initial bacterial concentration, DT is a function of the generation time and the length of the lag phase.

Genetic and biochemical characterization of mutants. Quinolone resistance mutations in the gyrA and parC genes of mutants selected in vitro or in vivo from parent strains BN18 and BN82 had earlier been identified by sequencing (Giraud et al., 1999). The persistence of mutations at codons 81,83 and 87 of gyrA was investigated, in mutants evolved either in vivo in the gut of chickens or in liquid culture, using the previously described AS-PCR-RFLP assay (Giraud et al., 1999). The presence of parC mutations at codon 80 of these evolved mutants was investigated by PCR-RFLP as described previously (Giraud et al., 1999).

Analysis of outer-membrane proteins by SDS-PAGE and immunoblot analysis of AcrA using an anti-AcrA polyclonal antibody were performed as described previously (Giraud et al., 2000).

Electron microscopy. Cells were grown to stationary phase in LB medium, harvested by centrifugation and resuspended in PBS at a concentration of about $10^{10} \mathrm{cells} \mathrm{ml}^{-1}$. An aliquot (5 $\mu \mathrm{l}$ ) of the suspension was applied for 2 min to Formvar, carbon-stabilized copper grids. Negative staining was performed with phosphotungstic acid for $1 \mathrm{~min}$. The grids were observed with a Philips CM10 microscope under standard operating conditions.

\section{RESULTS}

\section{Colonization experiments}

The ability to colonize the gut of chickens was tested for FQresistant mutants selected in vitro and in vivo from two susceptible strains. The mean levels of colonization achieved by parent strains $\mathrm{BN} 18$ and $\mathrm{BN} 82$ appeared stable for 4 weeks after the inoculation, at $10^{6}-10^{7}$ c.f.u. $\left(\mathrm{g}\right.$ faeces $\left.^{-1}\right)$. In contrast, the two highly ciprofloxacin-resistant in vitro mutants BN18/71 and BN82/71 (MIC respectively 8 and $16 \mu \mathrm{g} \mathrm{ml}^{-1}$ ) were detected only in samples of faeces taken at day 7 post-inoculation, and only after enrichment, indicating a level below $10^{2}$ c.f.u. $\left(\mathrm{g}\right.$ faeces ${ }^{-1}$ ). After 7 days, they were no longer detected. Thus, they did not colonize the gut of chickens permanently (Fig. 1). The in vivo-selected mutants, which exhibited intermediate resistance to ciprofloxacin (MIC $2 \mu \mathrm{g} \mathrm{ml}^{-1}$ ), colonized the gut of chickens at mean levels similar to (mutant BNJ-529) or significantly lower than (mutant BNJ-548) those reached by their respective parent strains (Fig. 1).

\section{Fitness and morphology of in vitro-selected mutants}

We had previously noted that highly ciprofloxacin-resistant mutants formed small colonies (Giraud et al., 1999). These growth defects were confirmed by generation-time determinations in liquid media: mutants BN18/71 and BN82/71 had altered growth profiles (Fig. 2), characterized by generation times 1.4- and 2-fold longer than their parent strains (Table 1). Detection times obtained using the Bactometer were also 1.3- and 2.2-fold longer for mutants BN18/71 and BN82/71 than for their parent strains. All the mutants achieved the

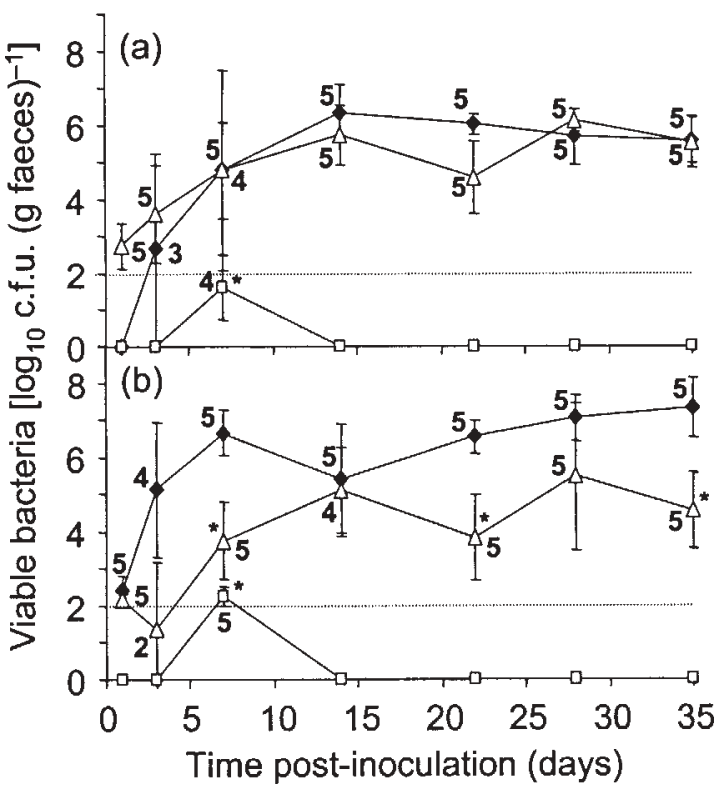

Fig. 1. Levels of colonization of the gut of chickens, evaluated from the number of viable bacteria recovered per gram faeces. Numbers next to each point indicate the number of animals positive out of five sampled. Asterisks indicate a level of colonization significantly lower $(P<0.002)$ than that reached by the parent strain. The horizontal line indicates the lower limit of detection. (a) Parent strain BN18 $(\bullet)$ and derived mutants BN18/71 $(\square)$ and BNJ-529 ( $\triangle$ ). (b) Parent strain BN82 $(\diamond)$ and derived mutants BN82/71 ( $\square$ ) and BNJ-548 ( $\triangle$ ).

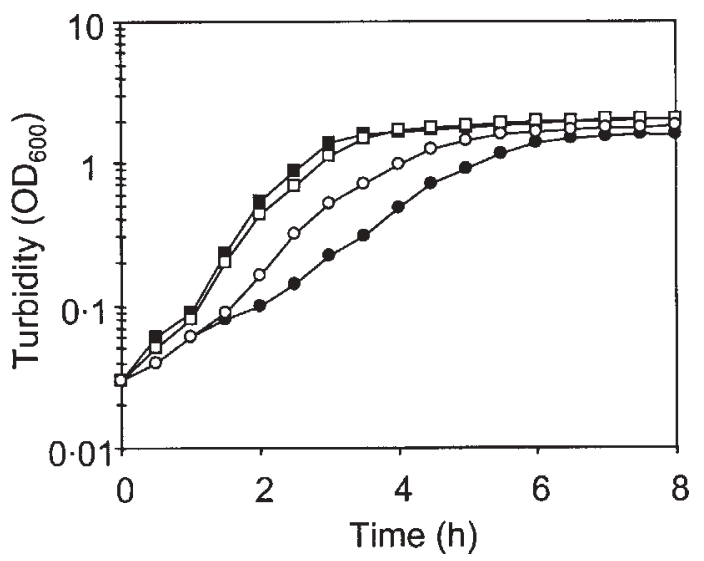

Fig. 2. Growth profiles of parent strains $B N 18(\square)$ and $B N 82(\square)$ and of their respective ciprofloxacin-resistant mutants BN18/71 (O) and BN82/71 (O)

same cell density as the parent strains at stationary phase. Viable cell counts did not reveal increased mortality (not shown).

Transmission electron microscopy was used to analyse the shape of cells of the highly ciprofloxacin-resistant mutant BN18/71. When grown to stationary phase, cells of the parent strain BN18 were long $(1 \cdot 5-3 \mu \mathrm{m})$ and thin $(0 \cdot 4 \mu \mathrm{m}$ in 
diameter) rods, whereas the mutant cells appeared as much shorter and thicker rods $(1.5 \mu \mathrm{m}$ long, $0.7 \mu \mathrm{m}$ in diameter $)$ (Fig. 3).

\section{Evolution of fitness}

The mutants selected in vivo, BNJ-529 and BNJ-548, also formed smaller colonies than their respective parent strains on solid medium (Giraud et al., 1999). On and after the third week of the colonization experiment, we observed two different types of colonies in plated samples of faeces from chickens contaminated with mutants BNJ-529 and BNJ-548: small colonies that looked like those formed by the inoculated mutant and fast-growing colonies that rapidly displayed a black centre on SS medium due to the production of $\mathrm{H}_{2} \mathrm{~S}$ (Fig. 4). The relative frequency of the fast-growing colonies increased until the end of the experiment. We determined the generation times of the two inoculated mutants (BNJ-529, BNJ-548) and of two clones that formed fast-growing colonies after passage in chickens. Surprisingly, the differences in growth rates seen on solid medium were not corroborated by generation time values (Table 1). However, the Bactometer experiments revealed significantly longer DTs (respectively $1 \cdot 2$ - and $1 \cdot 7$-fold) for mutants BNJ529 and BNJ-548 than for their parent strains. This observation suggests that these mutants could have longer lag times, which could also explain the smaller colonies on solid medium.

We also tested the ability of the highly ciprofloxacin-resistant in vitro mutants BN18/71 and BN82/71, which also formed micro-colonies, to recover higher growth rates spontaneously when serially passaged in a liquid medium without antibiotic. We maintained the two mutants in serial transfer culture for about 500 generations. The clones recovered after 500 generations still formed micro-colonies. The generation time of mutant BN18/71-R2 was reduced compared with that

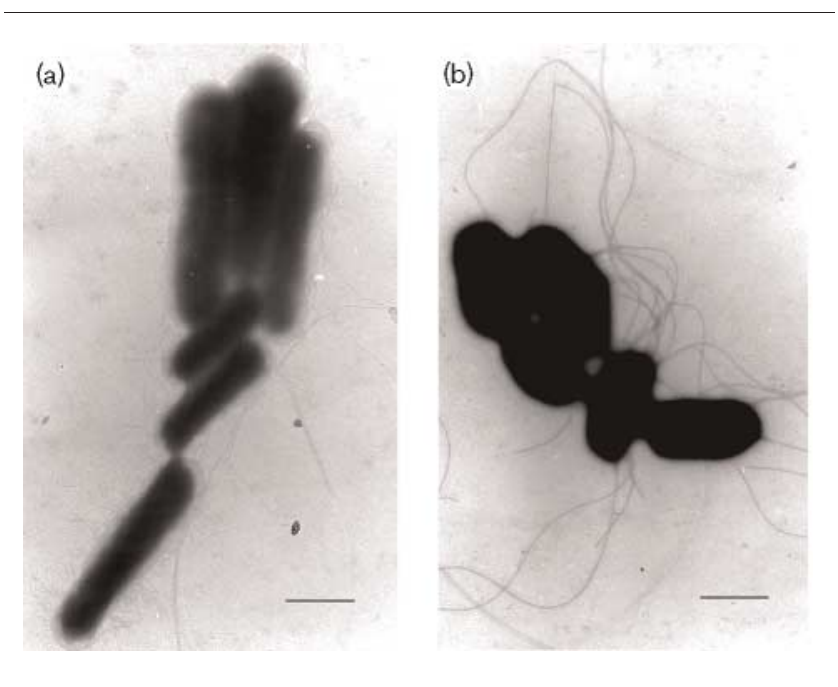

Fig. 3. Electron micrographs of cells of parent strain BN18 (a) and of ciprofloxacin-resistant mutant BN18/71 (b) grown to stationary phase. Bars, $1 \mu \mathrm{m}$.

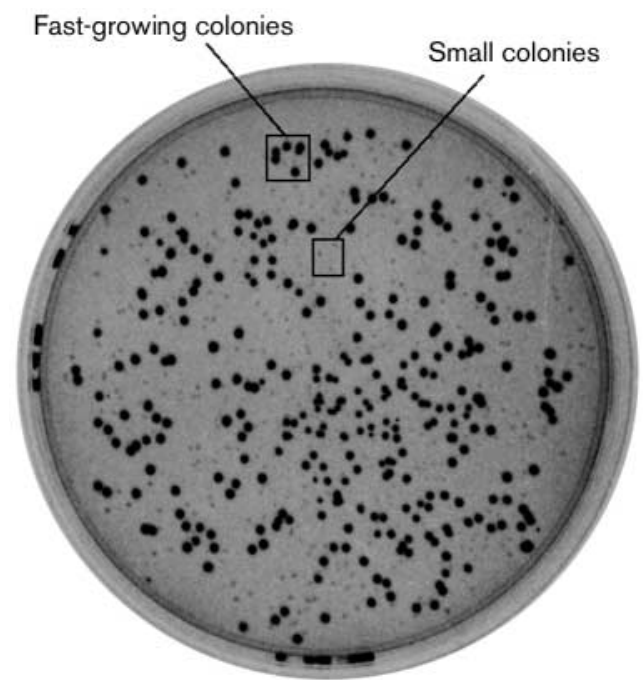

Fig. 4. Colonies formed by salmonellae present in the faeces of chickens 35 days after inoculation of mutant BNJ-548.

of mutant BN18/71, but was still longer than that of the susceptible parent strain BN18. The generation times of mutants BN82/71 and BN82/71-R2 were similar.

\section{Evolution of antibiotic susceptibilities}

The susceptibilities of mutants evolved either in vivo in the gut of chickens or in liquid culture to quinolones and unrelated antibiotics were close to those of the original mutants (Table 1). However, a slight decrease in resistance was noted for some evolved mutants, generally more pronounced for the older quinolone flumequin than for the newer FQs enrofloxacin and ciprofloxacin. The increase in susceptibility was greater for mutants derived from parent strain BN82 than for mutants derived from BN18. For BNJ$548 / \mathrm{G} 4$, the MICs of flumequin and ciprofloxacin were respectively 4 - and 2 -fold lower than for the originally inoculated mutant BNJ-548. For BN82/71-R2, the MICs of flumequin and enrofloxacin were 4 -fold lower than for BN82/71, and the MIC of ciprofloxacin was 2-fold lower. The MICs of tetracycline and chloramphenicol were 2 -fold lower in mutant BN18/71-R2, which could suggest that nonspecific resistance mechanisms such as active efflux may participate in the phenotype of resistance (Giraud et al., 2000).

\section{Topoisomerase mutations, AcrAB and porin expression}

We had shown previously that mutants selected in vitro or in vivo from parent strains $\mathrm{BN} 18$ and $\mathrm{BN} 82$ carried single or double mutations in the QRDR motif of gyrA, but did not show mutations in the parC QRDR (Giraud et al., 1999). All the derived mutants had conserved the gyrA mutations that confer quinolone resistance (Table 1). They did not have any mutation at codon 80 of parC. 
Since we have shown previously that the phenotype of resistance to quinolone was strongly correlated with the level of expression of the AcrAB efflux pump (Giraud et al., 2000), we tested whether the slight decrease in resistance observed for some of the mutants passaged in vitro or in chickens could be linked to a decreased expression of AcrAB. This possibility was also suggested by the slight decrease in resistance to structurally unrelated compounds like tetracycline or chloramphenicol (Table 1). Immunoblotting experiments showed that $\mathrm{Acr} A \mathrm{~B}$ was overexpressed in mutants selected both in vivo and in vitro, but no clear variation in $\mathrm{Acr} A \mathrm{~B}$ expression levels was detected after in vivo or in vitro passages (not shown). SDS-PAGE analysis of the outer-membrane proteins of all the mutants derived from parent strain BN18 did not reveal any modification of porin expression (not shown).

\section{DISCUSSION}

Whereas it is clear that genotypes resistant to an antibiotic are selectively favoured in the presence of this antibiotic, they often have lower growth rates than susceptible genotypes in an antibiotic-free environment. Such resistant genotypes should be rapidly counter-selected when placed in competition with susceptible counterparts or other species that occupy the same ecological niche and grow faster. However, it has been shown that resistant bacteria can restore their fitness partially or totally by accumulating compensatory mutations without losing their resistance (Andersson \& Levin, 1999; Gillespie \& McHugh, 1997; Lenski, 1997; Spratt, 1996). These compensatory mutations could prevent reversion to susceptibility not only by reducing the cost of resistance but also by creating a genetic background that is specifically adapted to resistance. For example, in Escherichia coli mutants resistant to streptomycin through the presence of mutations in rpsL, the accumulation of 'second-site' compensatory mutations generates a genetic background in which wild, susceptible alleles of $r p s L$ have an important selective disadvantage (Schrag et al., 1997).

Resistance to quinolones is also due to mutations in chromosomal genes such as those encoding the type II topoisomerase or those regulating the expression of multidrug efflux pumps. It can be speculated that the presence of mutations in these structural or regulatory genes, which are implicated in numerous cellular processes, not only increases resistance to quinolones but also affects the physiology of the bacterial cell. The fitness cost associated with quinolone resistance has not yet been studied extensively. A recent study showed that some, but not all, highly ciprofloxacin-resistant Escherichia coli isolates have altered growth rates (Bagel et al., 1999). However, it is not possible to know from this study whether acquisition of FQ resistance does not affect the fitness of Escherichia coli strains systematically or whether resistant isolates with normal growth rates have already restored their fitness by accumulating compensatory mutations. Bjorkman et al. (1998) found that chromosomal mutations that confer resistance to nalidixic acid, streptomycin or rifampicin decrease the competitive performance of $S$. Typhimurium in mice, but that compensatory mutations, rather than a true reversion to the sensitive wild-type, rapidly accumulate and restore virulence. They also demonstrated, in the case of resistance to streptomycin or to fusidic acid, that different fitness-compensating mutations are selected depending on whether the bacteria evolve through serial passage in mice or in a laboratory culture medium (Bjorkman et al., 2000).

Salmonellae apparently do not reach high levels of resistance by acquiring mutations successively and alternately in $g y r A$ and parC (Giraud et al., 1999). They may have no other means to face up to a high concentration of FQ than to use non-specific resistance mechanisms, such as active efflux by multidrug efflux pumps, at levels that would not be compatible with optimal growth. We have shown previously that the resistance phenotype of mutants derived from strain BN18 was strongly correlated with the level of expression of the AcrAB efflux pump, which was increased 10-fold in mutant BN18/71 (Giraud et al., 2000). Growth defects may be caused by the inopportune efflux of key metabolites by highly overexpressed multidrug pumps (Nikaido, 1998). However, possibly due to a lack of sensitivity of the immunoblot method, we did not detect any correlation between the slight decrease in resistance to quinolones and other unrelated antibiotics after in vitro or in vivo passage and decreased expression of AcrAB. Possible variations in the levels of expression of other multidrug efflux systems might also explain the small variations observed in resistance.

In the present study, we show that important physiological alterations accompany the acquisition of a high level of resistance to FQ by $S$. Typhimurium strains. These alterations, which find expression in decreased growth rates, the inability to colonize the gut of chickens and modified morphology, may explain why highly resistant mutants can be obtained by experimental selection in laboratory culture, but not under in vivo conditions, even in the absence of a competitive flora (Giraud et al., 1999). For our highly resistant mutants, no significant restoration of fitness and only a slight decrease in the level of resistance could be observed after a 500-generation evolution in an antibioticfree medium. For mutant BN82/71, the inability to colonize chickens and to regain fitness in the absence of FQ could be a consequence of the definitive loss of one or more porins that may also participate in the FQ-resistance phenotype of the mutant. For mutant BN18/71, this hypothesis can be excluded, since SDS-PAGE analysis has shown a porin profile identical to that of the parent strain BN18 (Giraud et al., 2000). This latter mutant, however, showed some alterations in outer-membrane proteins other than porins and of the LPS profile that could possibly account for the resistance, growth and colonization phenotypes. Mutants with intermediate resistance to ciprofloxacin (MIC $2 \mu \mathrm{g} \mathrm{ml}^{-1}$ ) successfully colonized the gut of chickens. We observed the emergence and propagation, in the gut of chickens, of subclones of these mutants that showed partially restored fitness and a slight decrease in resistance. One of these subclones had a resistance phenotype to quinolones that was 
close to those generally observed in nalidixic acid-resistant clinical isolates of Salmonella. On the basis of these results, we postulate that the infrequency of strains that are clinically resistant to ciprofloxacin could be due to the inability of most highly FQ-resistant $S$. Typhimurium mutants to compensate rapidly for their selective disadvantage before being excluded by faster-growing competitors that occupy the same ecological niche. As demonstrated by Bjorkman et al. (1998), nalidixic acid-resistant strains can restore their fitness by acquiring compensatory mutations. Our mutants, with an intermediate level of resistance to ciprofloxacin, apparently favoured a partial reversion to a level of susceptibility compatible with a normal growth rate, rather than the acquisition of compensatory mutations that would have maintained the level of resistance.

Although most clinical strains of $S$. enterica do not exhibit high-level resistance to FQ to date, recent reports demonstrate that some strains from different $S$. enterica serovars with high-level FQ resistance, combining stable and efficient multiple-target mutations in both $\operatorname{gra}$ and $\operatorname{parC}$ and probably also impermeability and efflux, can emerge and may be life-threatening (Chiu et al., 2002; Olsen et al., 2001; Nakaya et al., 2003). This strongly stresses the necessity of further surveillance of FQ resistance and the prudent use of FQ.

\section{ACKNOWLEDGEMENTS}

This work was supported by grants from the Ministère de la Recherche et de la Technologie. We thank J. C. Nicolle for his expert assistance in electron microscopy.

\section{REFERENCES}

Andersson, D. I. \& Levin, B. R. (1999). The biological cost of antibiotic resistance. Curr Opin Microbiol 2, 489-493.

Bagel, S., Hullen, V., Wiedemann, B. \& Heisig, P. (1999). Impact of gyrA and parC mutations on quinolone resistance, doubling time, and supercoiling degree of Escherichia coli. Antimicrob Agents Chemother $43,868-875$.

Baucheron, S., Imberechts, H., Chaslus-Dancla, E., Cloeckaert, A. (2002). The AcrB multidrug transporter plays a major role in high-level fluoroquinolone resistance in Salmonella enterica serovar Typhimurium phage type DT204. Microb Drug Resist 8, 281-289.

Bjorkman, J., Hughes, D. \& Andersson, D. I. (1998). Virulence of antibiotic-resistant Salmonella typhimurium. Proc Natl Acad Sci U S A 95, 3949-3953.

Bjorkman, J., Nagaev, I., Berg, O. G., Hughes, D. \& Andersson, D. I. (2000). Effects of environment on compensatory mutations to ameliorate costs of antibiotic resistance. Science 287, 1479-1482.

Brée, A., Dho, M. \& Lafont, J. P. (1989). Comparative infectivity for axenic and specific-pathogen-free chickens of $\mathrm{O} 2$ Escherichia coli strains with or without virulence factors. Avian Dis 33, 134-139.

Breuil, J., Brisabois, A., Casin, I., Armand-Lefevre, L., Fremy, S. \& Collatz, E. (2000). Antibiotic resistance in salmonellae isolated from humans and animals in France: comparative data from 1994 and 1997. J Antimicrob Chemother 46, 965-971.

Chen, J. Y., Siu, L. K., Chen, Y. H., Lu, P. L., Ho, M. \& Peng, C. F. (2001).
Molecular epidemiology and mutations at gyrA and parC genes of ciprofloxacin-resistant Escherichia coli isolates from a Taiwan medical center. Microb Drug Resist 7, 47-53.

Chiu, C. H., Wu, T. L., Su, L. H., Chu, C., Chia, J. H., Kuo, A. J., Chien, M. S. \& Lin, T. Y. (2002). The emergence in Taiwan of fluoroquinolone resistance in Salmonella enterica serotype Choleraesuis. $N$ Engl J Med 346, 413-419.

Cloeckaert, A. \& Chaslus-Dancla, E. (2001). Mechanisms of quinolone resistance in Salmonella. Vet Res 32, 291-300.

Deguchi, T., Fukuoka, A., Yasuda, M. \& 7 other authors (1997). Alterations in the GyrA subunit of DNA gyrase and the ParC subunit of topoisomerase IV in quinolone-resistant clinical isolates of Klebsiella pneumoniae. Antimicrob Agents Chemother 41, 699-701.

Gillespie, S. H. \& McHugh, T. D. (1997). The biological cost of antimicrobial resistance. Trends Microbiol 5, 337-339.

Giraud, E., Brisabois, A., Martel, J. L. \& Chaslus-Dancla, E. (1999). Comparative studies of mutations in animal isolates and experimental in vitro- and in vivo-selected mutants of Salmonella spp. suggest a counterselection of highly fluoroquinolone-resistant strains in the field. Antimicrob Agents Chemother 43, 2131-2137.

Giraud, E., Cloeckaert, A., Kerboeuf, D. \& Chaslus-Dancla, E. (2000). Evidence for active efflux as the primary mechanism of resistance to ciprofloxacin in Salmonella enterica serovar Typhimurium. Antimicrob Agents Chemother 44, 1223-1228.

Hansen, H., Rabsch, W., Heisig, P. (2001). Effect of gemifloxacin on in vitro selected mutants and field isolates of Salmonella spp. with mutations in $g y r A, \operatorname{gyr} B$, parC and marR. Supplementum 7th International Symposium on New Quinolones, abstract P80. J Antimicrob Chemother 47, 37.

Heisig, P. (1993). High-level fluoroquinolone resistance in a Salmonella typhimurium isolate due to alterations in both $\mathrm{gyrA}$ and $\mathrm{gyrB}$ genes. J Antimicrob Chemother 32, 367-377.

Heisig, P., Kratz, B., Halle, E., Graser, Y., Altwegg, M., Rabsch, W. \& Faber, J. P. (1995). Identification of DNA gyrase A mutations in ciprofloxacin-resistant isolates of Salmonella typhimurium from men and cattle in Germany. Microb Drug Resist 1, 211-218.

Lenski, R. E. (1997). The cost of antibiotic resistance - from the perspective of a bacterium. Ciba Found Symp 207, 131-140.

McDonald, L. C., Chen, F. J., Lo, H. J., Yin, H. C., Lu, P. L., Huang, C. H., Chen, P., Lauderdale, T. L. \& Ho, M. (2001). Emergence of reduced susceptibility and resistance to fluoroquinolones in Escherichia coli in Taiwan and contributions of distinct selective pressures. Antimicrob Agents Chemother 45, 3084-3091.

Members of the SFM Antibiogram Committee (2003). Comité de l'Antibiogramme de la Société Française de Microbiologie report 2003. Int J Antimicrob Agents 21, 364-391.

Molbak, K., Baggesen, D. L., Aarestrup, F. M., Ebbesen, J. M., Engberg, J., Frydendahl, K., Gerner-Smidt, P., Petersen, A. M. \& Wegener, H. C. (1999). An outbreak of multidrug-resistant, quinolone-resistant Salmonella enterica serotype Typhimurium DT104. N Engl J Med 341, $1420-1425$.

Nakaya, H., Yasuhara, A., Yoshimura, K., Oshihoi, Y., Izumiya, H. \& Watanabe, H. (2003). Life-threatening infantile diarrhea from fluoroquinolone-resistant Salmonella enterica Typhimurium with mutations in both gyrA and parC. Emerg Infect Dis 9, 255-257.

NCCLS (1997). Methods for dilution antimicrobial susceptibility tests for bacteria that grow aerobically, 4th edn. Approved Standards M7-A4. Wayne, PA: National Committee for Clinical Laboratory Standards.

Nikaido, H. (1998). Multiple antibiotic resistance and efflux. Curr Opin Microbiol 1, 516-523. 
Noble, A. P., Dziuba, M., Harrison, D. J., Albritton, W. L. (1999). Factors influencing capacitance-based monitoring of microbial growth. J Microbiol Methods 37, 51-64.

Olsen, S. J., DeBess, E. E., McGivern, T. E. \& 7 other authors (2001). A nosocomial outbreak of fluoroquinolone-resistant Salmonella infection. N Engl J Med 344, 1572-1579.

Paterson, D. L., Mulazimoglu, L., Casellas, J. M. \& 8 other authors (2000). Epidemiology of ciprofloxacin resistance and its relationship to extended-spectrum beta-lactamase production in Klebsiella pneumoniae isolates causing bacteremia. Clin Infect Dis 30, 473-478.

Robert, J., Cambau, E., Grenet, K., Trystram, D., Pean, Y., Fievet, M. H. \& Jarlier, V. (2001). Trends in quinolone susceptibility of Enterobacteriaceae among inpatients of a large university hospital: 1992-98. Clin Microbiol Infect 7, 553-561.

Schrag, S. J., Perrot, V. \& Levin, B. R. (1997). Adaptation to the fitness costs of antibiotic resistance in Escherichia coli. Proc R Soc Lond B Biol Sci 264, 1287-1291.
Spratt, B. G. (1996). Antibiotic resistance: counting the cost. Curr Biol 6, 1219-1221.

Threlfall, E. J., Ward, L. R., Skinner, J. A. \& Graham, A. (2000). Antimicrobial drug resistance in non-typhoidal salmonellas from humans in England and Wales in 1999: decrease in multiple resistance in Salmonella enterica serotypes Typhimurium, Virchow, and Hadar. Microb Drug Resist 6, 319-325.

Walker, R. A., Lawson, A. J., Lindsay, E. A. \& 7 other authors (2000). Decreased susceptibility to ciprofloxacin in outbreak-associated multiresistant Salmonella typhimurium DT104. Vet Rec 147, 395-396.

Weigel, L. M., Steward, C. D. \& Tenover, F. C. (1998). gyrA mutations associated with fluoroquinolone resistance in eight species of Enterobacteriaceae. Antimicrob Agents Chemother 42, 2661-2667.

Wiuff, C., Madsen, M., Baggesen, D. L. \& Aarestrup, F. M. (2000). Quinolone resistance among Salmonella enterica from cattle, broilers, and swine in Denmark. Microb Drug Resist 6, 11-17. 